\title{
Mycotic aneurysm of the aorta as a complication of Bacillus Calmette-Guérin instillation
}

\author{
${ }^{1} \mathrm{~J}$ Maundrell, ${ }^{2 S}$ Fletcher, ${ }^{3} \mathrm{P}$ Roberts, ${ }^{4} \mathrm{~A}$ Stein, ${ }^{5} \mathrm{M}$ Lambie \\ ${ }^{1}$ Foundation Year 1 Trainee, General Medicine, Warwick Hospital, South Warwickshire; ${ }^{2}$ Consultant Nephrologist; ${ }^{3}$ Consultant Vascular \\ Surgeon; ${ }^{4}$ Consultant Nephrologist, Renal and Vascular Departments, University Hospitals of Coventry and Warwickshire; ${ }^{5}$ Clinical Research \\ Fellow, Institute of Science and Technology in Medicine, Keele University, Keele, Staffordshire, UK
}

ABSTRACT Bacillus Calmette-Guérin (BCG) is a live attenuated strain of Mycobacterium bovis that has been widely used for the treatment of superficial transitional cell carcinoma of the bladder. We describe a rare case of supra-renal mycotic aortic aneurysm secondary to BCG instillation in a 75-year-old male. Patients presenting with systemic symptoms post-instillation, possibly with an aneurysm, should raise suspicion of BCG dissemination, which requires early instigation of anti-mycobacterial drugs.

KEYWORDS Bacillus Calmette-Guérin, intravesical immunotherapy, mycobacteria, mycotic aneurysm
Correspondence to M Lambie, Department of Nephrology, University Hospital of North Staffordshire, Royal Infirmary, Princess Road, Stoke on Trent, Staffordshire ST4 7LN, UK

tel. +44 (0) 1902307999

e-mail lambiem@doctors.org.uk

DECLARATION OF INTERESTS No conflict of interests declared.

\section{CASE REPORT}

A 75-year-old Caucasian male presented with a I5-month history of night sweats, fever, dyspnoea, productive cough and $19 \mathrm{~kg}$ weight loss. His medical history revealed hypertension, dyslipidaemia, chronic kidney disease, localised non-Hodgkin's lymphoma (treated with radiotherapy 30 years earlier) and a 15 pack-year ([packs smoked per day] $\times$ [years as a smoker]) smoking history. Sixteen months before his presentation the patient was diagnosed with in situ transitional cell carcinoma of the bladder.This was treated by transurethral resection, and he commenced a six-week course of intravesical bacillus Calmette-Guérin (BCG) treatment. The patient had no history of tuberculosis.

One day after the initial BCG instillation the patient noted fever, lethargy and headaches. Maintenance BCG therapy was discontinued after the first instillation due to the severity of symptoms. Symptoms progressed to include night sweats, productive cough, nausea, flu-like symptoms and increasing shortness of breath. Nine months later he was extensively investigated with no diagnosis achieved. This included sputum for acid-fast bacteria stain, tuberculosis testing (T-SPOT.TB), multiple blood, urine and sputum microbiological samples and chest radiograph, all of which were negative. A computed tomography (CT) scan of the chest and abdomen was reported as unremarkable.

Symptoms persisted despite broad spectrum antibiotic and steroid therapy and the patient was admitted from the renal clinic 15 months after BCG instillation, due to the patient's insistence that his symptoms had not abated and dated from the BCG treatment. Clinical examination revealed normal observations and the respiratory, abdominal and cardiac systems were unremarkable.

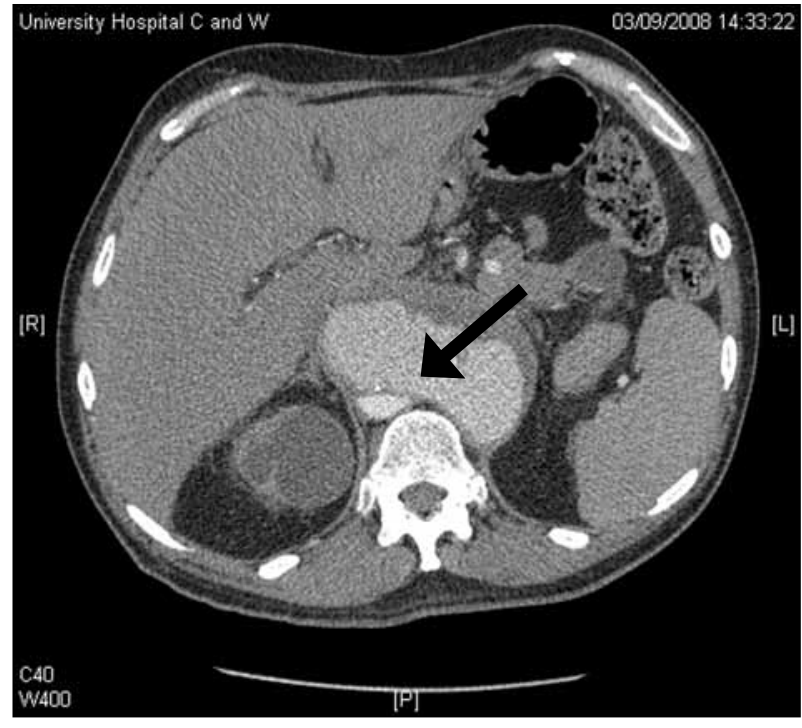

FIGURE I The patient's computed tomography scan of the abdomen identified a $5 \mathrm{~cm} \times 5 \mathrm{~cm} \times 10 \mathrm{~cm}$ soft tissue mass (arrow) affecting the supra-coeliac aorta, with an associated aneurysmal dilatation suggestive of mycotic aneurysm. Note the false lumen within the aneurysm.

Investigations demonstrated the haemoglobin to be $10.4 \mathrm{~g} / \mathrm{dl}$, and the C-reactive protein was raised at 40 $\mathrm{mg} / \mathrm{l}$, but the other routine tests were unremarkable. Immunological tests were unremarkable and all faecal, urine, blood, sputum and bone marrow cultures, including blood cultures incubated specifically for BCG, yielded no growth.

A CT scan of the abdomen (Figure I) identified a $5 \mathrm{~cm} \times$ $5 \mathrm{~cm} \times 10 \mathrm{~cm}$ soft tissue mass affecting the supra-coeliac aorta, with an associated aneurysmal dilatation suggestive of mycotic aneurysm. Retrospectively, some subtle changes were identified at the time of the first scan. 
Subsequent bone marrow aspirate and trephine biopsy revealed interstitial and paratrabecular granulomata with caseous necrosis, suggestive of mycobacterial infection. Ziehl-Neelsen and Periodic acid-Schiff staining and extended incubation of bone marrow cultures demonstrated no evidence of acid-fast bacteria or fungi.

A BCG-induced supra-renal mycotic aortic aneurysm was diagnosed.The patient was initiated on anti-mycobacterial chemotherapy comprising of rifampicin, isoniazid and ethambutol, and this resulted in symptom resolution. Five months later a repeat $\mathrm{CT}$ abdomen scan revealed a substantial increase in size of the aortic aneurysm, now measuring $10 \mathrm{~cm}$ in diameter and $8 \mathrm{~cm}$ longitudinally. At surgery an axillo bi-femoral graft was inserted and thoraco-laparotomy performed to explore the aneurysm. Renal artery involvement meant the repair could not be completed and the patient died of aortic aneurysm rupture one month later. Unfortunately, aortic aneurysm biopsies were not taken and an autopsy was not performed.

\section{DISCUSSION}

In this case, suggestive symptoms were temporally coincident with intravesical BCG therapy. This, coupled with caseating granulomata of the bone marrow, a negative tuberculosis test and a mycotic aneurysm, makes the diagnosis of 'BCG-osis' highly likely, despite the lack of definitive microbiological identification.

Bacillus Calmette-Guérin is a live attenuated strain of Mycobacterium bovis that has been used for the treatment of superficial transitional cell carcinoma of the bladder over the past three decades. Of those presenting with bladder cancer $70-80 \%$ are superficial transitional cell carcinomas (stage $\mathrm{Ta}-\mathrm{TI}$ or carcinoma in situ) for which standard treatment (transurethral resection followed by intravesical BCG immunotherapy) can be adopted. Intravesical BCG therapy is an effective prophylaxis in reducing the progression, number and frequency of tumour reccurrence. ${ }^{1,2}$

The most common symptoms ( $<5 \%$ of recipients) include dysuria, urinary frequency, haematuria, mild malaise and low-grade fever. Symptoms typically subside within 48 hours; more severe or prolonged cases may have a lower dose without reduction in efficacy. ${ }^{2,3}$ Severe side effects are infrequent but include systemic septic or hypersensitivity reactions with symptoms of chills, fever, hypotension and multi-organ dysfunction. Disseminated BCG infection is also reported, including epididymitis, hepatitis, pneumonitis and prostatitis. ${ }^{3}$

Bacillus Calmette-Guérin-induced mycotic aneurysms are rare, with only 15 previously documented cases since the introduction of intravesical BCG immunotherapy in 1974. .9 $^{4}$ The majority of infections occured within the abdominal aorta, three are reported in the femoral artery (two with concurrent abdominal aortic aneurysm) and one in the popliteal artery.

Infection from BCG instillation has been classified into early (within three months), predominantly hypersensitivity reactions, and late presentations ( $>$ I year), usually focal infections of the urinary tract. ${ }^{10}$ Early presentation is considered a systemic response to a low-grade pathogen in an immunologically competent host, in contrast to late presentation in which a reactivation of the infection occurs. Infected aneurysms have been reported in both early and late presentations. In our case, it is possible that the prominence of the underlying immunocompromise and lack of early treatment account for the early presentation.

Haematogenous dissemination through the vasa vasorum or lymphatic spread in the retroperitoneum following instillation is considered a plausible mechanism of infection. ${ }^{7}$ Risk factors pertaining to this are a breach of the uroepithelium facilitating microbial entry into the blood or lymphatic system. Delaying instillation for several weeks following gross haematuria, cystitis, catheterisation trauma or transurethral resection of bladder tumour are recommended."

The International Bladder Cancer Group recommends the permanent cessation of BCG therapy, plus the institution of two or more antimicrobial agents (fluoroquinolones, isoniazid, rifampicin), if high-grade fever persists $\left(>38.5^{\circ} \mathrm{C}\right.$ for $>48$ hours). "In severe systemic BCG reactions, a discontinuation of BCG therapy, initiation of high-dose fluoroquinolones and corticosteroids (based on animal models) and daily isoniazid, rifampicin and ethambutol for a duration of six months are advised. Bacillus Calmette-Guérin is not responsive to pyrazinamide.

For the treatment of the aneurysm, surgical intervention was adopted in all 16 aortic aneurysmal cases (including the present case), with concomitant anti-mycobacterial treatment implemented in II cases. Three deaths occurred, of which two cases failed to receive anti-mycobacterial treatment. The third case, presented here, received delayed treatment, but a more favourable outcome may have been achieved had treatment commenced earlier. According to Harding et al., 7 the mean delay from BCG administration to treatment is at least 23 months, demonstrating a clear need for greater awareness of this treatable, serious, iatrogenic condition.

\section{CONCLUSION}

In conclusion, intravesical BCG immunotherapy can be associated with systemic complications, including mycotic aneurysms. A high index of suspicion for disseminated $B C G$, including mycotic aneurysm, plus early instigation of anti-mycobacterial drugs and aneurysmal surgical repair is required in patients presenting with symptoms following intravesical BCG therapy. 


\section{REFERENCES}

I Han RF, Pan JG. Can intravesical Bacillus Calmette-Guérin reduce recurrence in patients with superficial bladder cancer? A metaanalysis of randomized trials. Urology 2006; 67:1216-23. doi:10.1016/j.urology.2005.12.014

2 Lockyer CR, Gillatt DA. BCG immunotherapy for superficial bladder cancer. J R Soc Med 200I; 94:II9-23.

3 Lamm DL. Efficacy and safety of bacille Calmette-Guérin immunotherapy in superficial bladder cancer. Clin Infect Dis 2000; 3I(Suppl 3):S86-90. doi: I0.1086/314064

4 Wolf YG, Wolf DG, Higginbottom PA et al. Infection of a ruptured aortic aneurysm and an aortic graft with bacille Calmette-Guérin after intravesical administration for bladder cancer. J Vasc Surg 1995; 22:80-4. doi:10.1016/S0741-52 14(95)70092-7

5 Damm OL, Briheim G, Hagstrom T et al. Ruptured mycotic aneurysm of the abdominal aorta: a serious complication of intravesical instillation bacillus Calmette-Guérin therapy. J Urol 1998; 159:984. doi: 10.1016/S0022-5347(0I)63796-0

6 Kamphuis JT, Buiting AG, Miseré JF et al. BCG immunotherapy: be cautious of granulomas. Disseminated BCG infection and mycotic aneurysm as late complications of intravesical BCG instillations. Neth J Med 200I; 58:7I-5. doi:I0.10I6/S0300-2977(00)00098-X
7 Harding GE, Lawlor DK. Ruptured mycotic abdominal aortic aneurysm secondary to Mycobacterium bovis after intravesical treatment with bacillus Calmette-Guérin. J Vasc Surg 2007; 46:I3 I-4. doi:10.1016/j.jvs.2007.01.054

8 Safdar N, Abad CL, Kaul DR et al. Clinical problem-solving: an unintended consequence. N Engl J Med 2008; 358:|496-50I. doi: I0.1056/NEJMcps07067I I

9 Costiniuk CT, Sharapov AA, Rose GW et al. Mycobacterium bovis abdominal aortic and femoral artery aneurysms following intravesical bacillus Calmette-Guérin therapy for bladder cancer. Cardiovasc Pathol 2010; 19:e29-32. doi:10.1016/j.carpath.2008.09.003

I0 Witjes JA, Palou J, Soloway $M$ et al. Clinical practice recommendations for the prevention and management of intravesical therapyassociated adverse events. Eur Urol Suppl 2008; 7:667-74. doi:10.1016/j.eursup.2008.08.001

\section{INVITATION TO SUBMIT PAPERS}

We would like to extend an invitation to all readers of The Journal of the Royal College of Physicians of Edinburgh to contribute original material, especially to the clinical section. The JRCPE is a peer-reviewed journal with a circulation of 8,000 . Its aim is to publish a range of clinical, educational and historical material of crossspecialty interest to the College's international membership.

The JRCPE is currently indexed in Medline, Embase, Google Scholar and the Directory of Open Access Journals. The editorial team is keen to continue to improve both the quality of content and its relevance to clinical practice for Fellows and Members. All papers are subject to peer review and our turnaround time for a decision averages only eight weeks.

We would be pleased to consider submissions based on original clinical research,

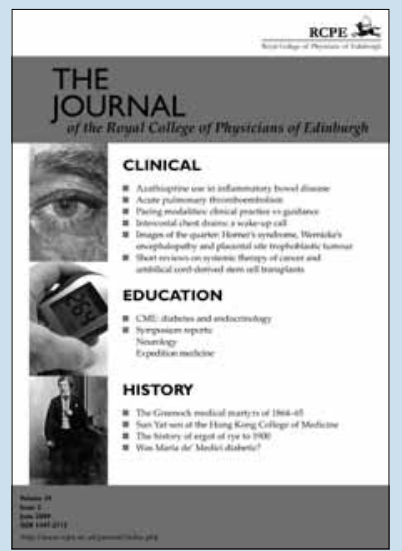
including pilot studies. The JRCPE is a particularly good forum for research performed by junior doctors under consultant supervision. We would also consider clinical audits where the 'loop has been closed' and a demonstrable clinical benefit has resulted.

For further information about submissions, please visit: http://www.rcpe.ac.uk/journal/contributers.php or e-mail editorial@rcpe.ac.uk.Thank you for your interest in the College's journal.

The editorial team,

The Journal of the Royal College of Physicians of Edinburgh 